\title{
Stringent neutron-star limits on large extra dimensions
}

\author{
Steen Hannestad \\ NORDITA, Blegdamsvej 17, 2100 Copenhagen, Denmark \\ Georg G. Raffelt \\ Max-Planck-Institut für Physik (Werner-Heisenberg-Institut), Föhringer Ring 6, 80805 München, Germany
}

(12 December 2001)

\begin{abstract}
Supernovae (SNe) are copious sources for Kaluza-Klein gravitons which are generic for theories with large extra dimensions. These massive particles are produced with average velocities $\simeq 0.5 c$ so that many of them are gravitationally retained by the SN core. Every neutron star thus has a halo of KK gravitons which decay into $\nu \bar{\nu}, e^{+} e^{-}$and $\gamma \gamma$ on time scales $\simeq 10^{9}$ years. The EGRET $\gamma$-flux limits $\left(E_{\gamma} \simeq 100 \mathrm{MeV}\right)$ for nearby neutron stars constrain the compactification scale for $n=2$ extra dimensions to $M \gtrsim 500 \mathrm{TeV}$, and $M \gtrsim 30 \mathrm{TeV}$ for $n=3$. The requirement that neutron stars are not excessively heated by KK decays implies $M \gtrsim 1700 \mathrm{TeV}$ for $n=2$, and $M \gtrsim 60 \mathrm{TeV}$ for $n=3$.
\end{abstract}

PACS numbers: 11.10.Kk, 98.70.Vc, 12.10.-g

Introduction.-Theories with large extra dimensions are a new alternative to solve the hierarchy problem of particle physics [1 5]. Apart from cosmology [6, 7], the most restrictive limits on the size of the extra dimensions derive from astrophysical arguments. The existence of Kaluza-Klein gravitons, particles with an essentially continuous spectrum of masses, is a generic feature of the new theory. Even though the KK states couple with the strength of ordinary gravitons, the large number of modes implies that stars are copious sources for these particles [8]. Until recently, the most restrictive limit derived from the requirement that the SN 1987A neutrino signal was not unduly shortened by the new energy loss, i.e. that the fraction of energy carried away by KK gravitons obeys $f_{\mathrm{KK}} \lesssim 0.5[9] 12$. For $n=2$ or 3 extra dimensions, this constraint translates into limits on the compactification scale $M$ summarized in Table [1. (One extra dimension is already excluded by other arguments.)

We recently obtained much more restrictive limits when taking KK graviton decays into account 13]. Most of the KK states are produced with masses near the kinematical threshold. For a SN core with $T \simeq 30 \mathrm{MeV}$ this implies $m \simeq 100 \mathrm{MeV}$ so that the only decay channels are $\nu \bar{\nu}, e^{+} e^{-}$and $\gamma \gamma$ with $\tau_{2 \gamma}=\frac{1}{2} \tau_{e^{+} e^{-}}=\tau_{\nu \bar{\nu}} \simeq$ $6 \times 10^{9} \mathrm{yr}(\mathrm{m} / 100 \mathrm{MeV})^{-3}$ 迎. The cosmic $\gamma$-ray background measured by the EGRET satellite then yields the limits shown in Table 1 , using all SNe in the universe as sources. Of course, this argument depends on the assumption that there are no fast invisible decay channels into other KK excitations (this could be the case for nontoroidal compactification [14]), and also that the graviton emission is not suppressed as in some models [15].

We presently show that even more restrictive constraints follow from the EGRET $\gamma$-ray limits for young nearby SN remnants and nearby neutron stars. Taking Cas A $(3.4 \mathrm{kpc})$ as a first example, the limits are compa- rable to the cosmic case. The key ingredient is that even 320 years after the explosion the decay photons come from the direction of Cas A within the EGRET angular resolution. For SN 1987A the limits are less restrictive because of its relatively large distance of $50 \mathrm{kpc}$.

The kinetic energy of the thermally produced KK gravitons is small so that a large fraction of those produced in the hot inner SN core remain gravitationally trapped. Therefore, every neutron star is surrounded by a halo of KK gravitons which is dark except for the decays into $\simeq 100 \mathrm{MeV}$ neutrinos, $e^{+} e^{-}$pairs and $\gamma$-rays. Since neutron stars are observed as close as 60 pc, one gains a huge flux factor relative to Cas A.

These arguments imply that future $\gamma$-ray telescopes such as GLAST could be in a position to find a signature for KK graviton decays from nearby neutron stars.

However, this possibility is marginal in view of yet more restrictive limits. The KK decays in and around neutron stars provide a heat source which prevents it cooling below a level where its thermal emission is com-

TABLE I. Constraints from $\mathrm{SNe}$ and neutron stars.

\begin{tabular}{|c|c|c|c|c|}
\hline \multirow[t]{2}{*}{ Observation \& Object } & \multicolumn{2}{|c|}{$f_{\mathrm{KK}}^{\max }$} & \multicolumn{2}{|c|}{$M^{\min }[\mathrm{TeV}]$} \\
\hline & $n=2$ & $n=3$ & $n=2$ & $n=3$ \\
\hline \multicolumn{5}{|l|}{ Neutrino signal } \\
\hline SN 1987A 12 & 0.5 & 0.5 & 31 & 2.75 \\
\hline \multicolumn{5}{|l|}{ EGRET $\gamma$-ray limits } \\
\hline Cosmic SNe 13] & $0.5 \times 10^{-2}$ & $0.5 \times 10^{-2}$ & 84 & 7 \\
\hline Cas A & $1.6 \times 10^{-2}$ & $0.6 \times 10^{-2}$ & 73 & 7 \\
\hline PSR J0953+0755 & $4.4 \times 10^{-5}$ & $1.8 \times 10^{-5}$ & 300 & 19 \\
\hline RX J185635-3754 & $1.0 \times 10^{-5}$ & $0.4 \times 10^{-5}$ & 454 & 27 \\
\hline \multicolumn{5}{|c|}{ Neutron star excess heat } \\
\hline PSR J0953+0755 & $0.5 \times 10^{-7}$ & $0.5 \times 10^{-7}$ & 1680 & 60 \\
\hline \multicolumn{5}{|c|}{ GLAST $\gamma$-ray sensitivity } \\
\hline RX J185635-3754 & $1 \times 10^{-7}$ & $0.5 \times 10^{-7}$ & 1300 & 60 \\
\hline
\end{tabular}


parable to the KK heating. The low measured luminosity of some pulsars then provides the most restrictive limits on large extra dimensions.

Supernova remnant Cas A.-This object probably corresponds to Flamsteed's SN of 1680. The CHANDRA x-ray satellite has unambiguously observed a non-pulsing thermal x-ray source in Cas A [16], a compact remnant, so that Cas A was indeed formed by a core-collapse event. At a distance of $3.4 \mathrm{kpc}$ it is the closest of the historical core-collapse SNe except for the Crab (2 kpc) which, however, is a strong EGRET source and as such not useful to place $\gamma$-ray limits on KK decays.

The EGRET experiment has made a full sky survey for $\gamma$-ray sources in the $30 \mathrm{MeV}-10 \mathrm{GeV}$ range [17. No source was detected close to the site of Cas A, implying a point-source flux limit of [18]

$$
\phi_{E>100 \mathrm{MeV}} \lesssim 10^{-7} \mathrm{~cm}^{-2} \mathrm{~s}^{-1} \text {. }
$$

Photons in this energy band would come from graviton decays with $m \gtrsim 200 \mathrm{MeV}$. Assuming a SN core temperature of $30 \mathrm{MeV}$, KK gravitons in this mass range would be produced with an average velocity of $\simeq 0.5 c$, and those which escape the gravitational potential would end up being much slower. Therefore, in 320 years the KK graviton cloud would have expanded no further than about $150 \mathrm{lyr}$, or an angular extent of $1.5^{\circ}$. Since the EGRET beam width at these energies is about $5^{\circ}$, the KK cloud is indeed equivalent to a point source.

The expected $\gamma$-ray flux from this cloud is written as

$$
\begin{aligned}
\frac{d \phi}{d z} & =2 f_{\mathrm{KK}} \Gamma(z) \frac{E_{\mathrm{TOT}}}{\left\langle E_{\mathrm{KK}}\right\rangle} \frac{d N}{d z} \frac{1}{4 \pi d^{2}} \\
& =1.4 \times 10^{-2} \mathrm{~cm}^{-2} \mathrm{~s}^{-1} f_{\mathrm{KK}} d_{\mathrm{kpc}}^{-2} T_{30}^{2} \beta^{-1} z^{3} \frac{d N}{d z},
\end{aligned}
$$

where $z=E_{\gamma} / T$ with $T$ the average temperature of the protoneutron star and $\Gamma(z)$ the decay rate of gravitons with the mass $m=2 E_{\gamma}$. $E_{\text {TOT }}$ is the total energy emitted by the SN, taken to be $3 \times 10^{53} \mathrm{erg}$, and $f_{\mathrm{KK}}$ the fraction emitted in the form of $\mathrm{KK}$ gravitons. $\left\langle E_{\mathrm{KK}}\right\rangle$ is the mean KK energy, $\frac{d N}{d z}$ the normalized photon distribution, and $d$ the distance to the source. Further, $T_{30}=T / 30 \mathrm{MeV}$ and $\beta=\left\langle E_{\mathrm{KK}}\right\rangle / T$, where $\beta=4.25$ for $n=2$ and $\beta=5.42$ for $n=3$. We neglect the kinetic energy of the KK gravitons so that $E_{\gamma}=m / 2$, and we also neglect the Lorentz factor in the decay lifetime. These approximations have no significant impact on our results. The relevant emission process is nucleon bremsstrahlung $N+N \rightarrow N+N+$ KK [1].

Assuming an average temperature $T=30 \mathrm{MeV}$ for the SN core, the $\gamma$ luminosity of the KK cloud of Cas A is about $f_{\mathrm{KK}} 1.0 \times 10^{4} L_{\odot}$ for $n=2$ and $f_{\mathrm{KK}} 2.0 \times 10^{4} L_{\odot}$ for $n=3$. The expected $\gamma$ flux at Earth is

$$
\phi_{E>100 \mathrm{MeV}}=f_{\mathrm{KK}} \times \begin{cases}6.0 \times 10^{-6} \mathrm{~cm}^{-2} \mathrm{~s}^{-1} & \text { for } n=2, \\ 1.6 \times 10^{-5} \mathrm{~cm}^{-2} \mathrm{~s}^{-1} & \text { for } n=3 .\end{cases}
$$

Comparing this with the EGRET limit of Eq. (11) constrains $f_{\mathrm{KK}}$ and the compactification scale at a comparable level to the cosmic $\mathrm{SNe}$ (Table II).

Our bounds on the compactification scale are quite robust because they vary with the KK emission limit as

$$
M^{\min } \propto\left(f_{\mathrm{KK}}^{\max }\right)^{-1 /(n+2)} .
$$

Therefore, $M^{\text {min }}$ depends only very weakly on the exact value of the EGRET flux limit or on the assumed SN properties.

Neutron Stars. - The gravitational potential at the surface of a neutron star with mass $M$ and radius $R$ is $\Phi_{R}=-G_{\mathrm{N}} M / R=-0.154\left(M / M_{\odot}\right)(10 \mathrm{~km} / R)$. The escape velocity from this location is

$$
v_{\mathrm{esc}}=\sqrt{-2 \Phi_{R}}=0.55\left(\frac{M / M_{\odot}}{R / 10 \mathrm{~km}}\right)^{1 / 2} .
$$

This is similar to the average speed of the thermally produced KK gravitons so that a SN core retains a large fraction of these particles within a halo of size a few $R$. This halo will continue to shine in $\gamma$-rays from KK decay even a long time after the original SN explosion.

In order to calculate the expected flux we use Eq. (2), taking into account two additional factors. One is $F(z)$, the fraction of KK-gravitons trapped close to the neutron star, which depends on the KK-mass and thus on $z$. To calculate $F(z)$ we used $R=12 \mathrm{~km}$ and $M=1.4 M_{\odot}$, corresponding to an escape velocity of $0.59 \mathrm{c}$. In Fig. 1 we show $F(z)$ as a function of $z$. Note that it depends on $z$, but not on $n$. The differential energy-loss rate as a function of $z=E_{\gamma} / T$ was shown in Fig. 1 of [13]. Depending on $n$ it peaks for $z=2-4$, implying an average retention fraction of about $1 / 2$.

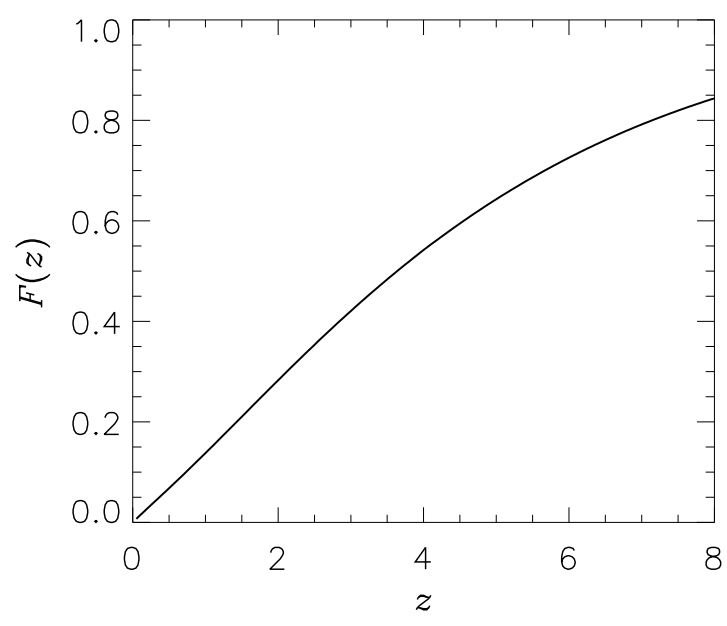

FIG. 1. The trapping fraction of KK-gravitons, $F(z)$, shown as a function of $z$. 
TABLE II. Constraints on $f_{K K}$ from nearby neutron stars.

\begin{tabular}{lrcccc}
\hline \hline Object & Ref. & $d$ & $t_{\mathrm{NS}}$ & \multicolumn{2}{c}{$f_{\mathrm{KK}}^{\max } \times 10^{5}$} \\
& & {$[\mathrm{kpc}]$} & {$\left[10^{6} \mathrm{yr}\right]$} & $n=2$ & $n=3$ \\
\hline RX J185635-3754 & 19 & 0.06 & 1.2 & 0.96 & 0.36 \\
PSR J0108-1431 & 20 & 0.1 & 160 & 11. & 4.8 \\
PSR J0953+0755 & 21 & 0.12 & 17 & 4.4 & 1.8 \\
PSR J1932+1059 & 22 & 0.17 & 3.1 & 7.6 & 3.0 \\
\hline \hline
\end{tabular}

A second effect is that for old pulsars many of the gravitons have decayed already, reducing the presentday source strength. Therefore, the second factor is $\exp \left(-t_{\mathrm{NS}} / \tau(z)\right)$ with $\tau(z)$ the total lifetime of the relevant KK mode and $t_{\mathrm{NS}}$ the age of the neutron star.

Based on this reasoning we show in Table II the limits on $f_{\mathrm{KK}}$ obtained from several nearby neutron stars. The closest and youngest case, RX J185635-3754, is a nonpulsing, thermal x-ray source. The parallax of the optical counterpart has been measured, yielding a distance of 60 pc. The closest EGRET source to this neutron star is $3 \mathrm{EG} \mathrm{J1847-3219}$ at an angular distance of about $6.2^{\circ}$, far enough away to allow for useful $\gamma$-ray limits. We have not used PSR J0437-4715 23 ( $d=0.14 \mathrm{kpc})$ because it is very old so that much of the original KK population would have decayed already.

In all cases we have assumed the EGRET point-source limit of Eq. (11). In practice, the exact limit depends on many factors, notably the source location relative to the galactic plane and thus on the diffuse background in the neighborhood of the relevant object. However, we really aim at a limit on the compactification scale which depends only weakly on $f_{\mathrm{KK}}$ - see Eq. (伍). Moreover, the limits from all of these neutron stars are comparable so that one need not rely on any particular case for an approximate overall constraint. For the two most restrictive cases we give our nominal limits on the compactification scale in Table 1 . These are by far the most restrictive limits on large extra dimensions.

The GLAST $\gamma$-ray satellite [24], to be launched in 2006, will have a point-source flux sensitivity of $\simeq 1.5 \times$ $10^{-9} \mathrm{~cm}^{-2} \mathrm{~s}^{-1}$. For the neutron star RX J185635-3754 this corresponds to a detection limit of $f_{\mathrm{KK}} \simeq 10^{-7}$ for $n=2$ and $\simeq 0.5 \times 10^{-7}$ for $n=3$. Using $f_{\mathrm{KK}}=10^{-7}$ we show in Fig. 2 2 the expected $\gamma$-ray flux if the average SN core temperature was $30 \mathrm{MeV}$.

Neutron star heating by KK-decays. - Several older, isolated neutron stars seem to have surface temperatures much higher than expected in standard cooling models [25,26]. The pulsars PSR J0953+0755 and PSR J1932+1059 have both been observed with the HST and radiation has been detected which is interpreted as almost thermal emission from the neutron star surface. In particular for PSR J0953+0755 the surface temperature is tightly constrained to be $T=(7 \pm 1) \times 10^{4} \mathrm{~K}$. Standard cooling models predict a much lower temperature

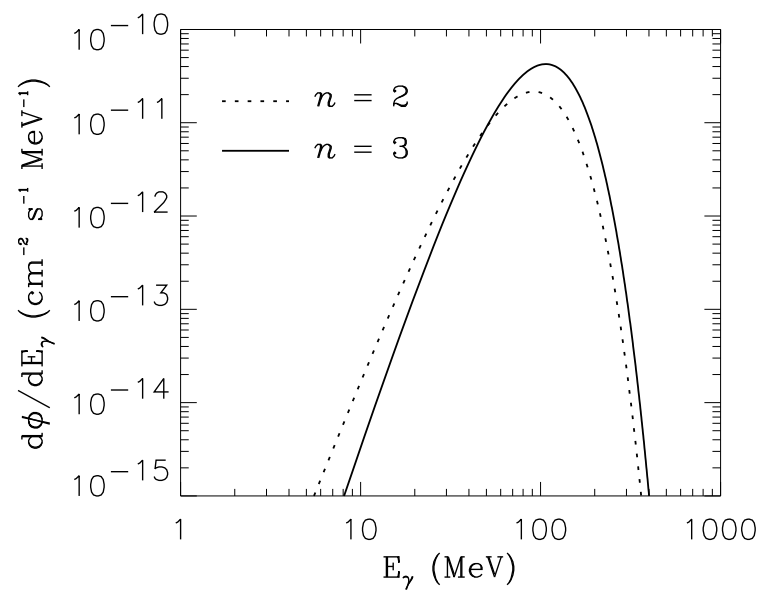

FIG. 2. The $\gamma$-flux from RX J185635-3754 for $f_{\mathrm{KK}}=10^{-7}$ and an assumed SN core temperature of $30 \mathrm{MeV}$.

for a neutron star of this age. Several models have been proposed to explain this excess heating which appears to be of order $10^{-5} L_{\odot}$ 26]. One promising explanation involves friction between the crust of the neutron star and the superfluid interior as an internal heat source.

However, the excess heat can also be generated by the cloud of KK-gravitons surrounding the neutron star. A flux of $\gamma$-rays, electrons, positrons, and neutrinos continuously hit the neutron star and heat it. (Note that a neutron star is not transparent for neutrinos in the $100 \mathrm{MeV}$ range. Note also that the charged particles may be channelled to the polar caps by magnetic fields.) In Fig. 3 we show the estimated heating rate from KKdecays as a function of the neutron star age. The heating rates plotted in the figure only include heating by

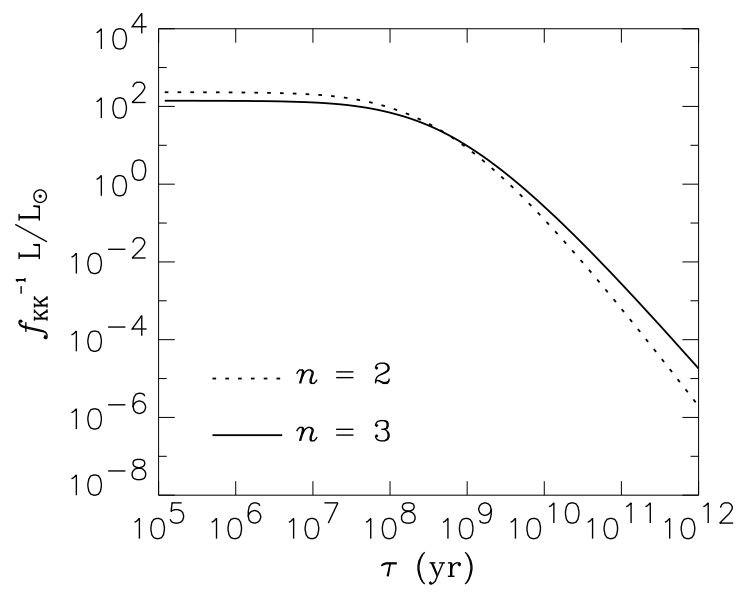

FIG. 3. The heating luminosity of a neutron star due to KK-decays as a function of age. 
gamma-rays, not electrons, positrons, and neutrinos, so that the true heating rate could be higher by a factor of a few. PSR J0953+0755 is quite young and the energy deposited on the neutron star is therefore $200-300 L_{\odot} f_{\mathrm{KK}}$. For the heating not to exceed the observed luminosity one would need to require $f_{\mathrm{KK}} \lesssim 5 \times 10^{-8}$, by far the most restrictive limit. We stress that the exact number is uncertain within a factor of a few, both because the calculation of neutron star heating by KK-decays involves some uncertainties, and because the temperature measurement of the neutron star could be more uncertain than the quoted error bars.

Interestingly, this result implies that if neutron-star heating is indeed due to KK-decays, then the relevant level of KK emission from a SN core is where the GLAST satellite may just about observe KK decays directly from RX J185635-3754.

We have not studied in detail the cases for $n \geq 4$ because we have relied on the SN emission calculations of 11, 12 for $n=2$ and 3. Simple scaling arguments suggest that for $n=4$ the neutron-star heating limit would be $M_{4}^{\min } \simeq 4 \mathrm{TeV}$ and $M_{5}^{\text {min }} \simeq 0.8 \mathrm{TeV}$.

Summary. - A large fraction of the KK gravitons produced by a SN core are gravitationally retained in a cloud surrounding the neutron star. The decay time of these particles is of order $10^{9}$ years, the dominant modes have masses of order $100 \mathrm{MeV}$. Therefore, neutron stars should shine brightly in $100 \mathrm{MeV} \gamma$ rays, contrary to observations with the EGRET satellite, allowing us to derive the most restrictive limits on large extra dimensions. In addition, the excess heating of neutron stars caused by the KK cloud prevents neutron stars from cooling below a certain temperature. The observed low luminosity of neutron stars provides even more restrictive limits on the compactification scale.

For $n=2$ our present limits $M>500-1600 \mathrm{TeV}$ put the compactification scale far above the weak unification scale. If large extra dimensions solve the hierarchy problem, $M$ should not exceed $10-100 \mathrm{TeV}$, a requirement which already excludes the $n=1$ case. Our new limits suggest that the $n=2$ case is also no longer plausible, and even $n=3$ now seems less appealing. Of course, our limits for $n>1$ only apply for a situation where all extra dimensions have the same compactification radius. In the general case the bounds could be much less restrictive.

Acknowledgments.-In Munich, this work was partly supported by the Deutsche Forschungsgemeinschaft under grant No. SFB 375 and the ESF network Neutrino Astrophysics. We thank Thomas Janka for calling our attention to the neutron star RX J185635-3754 and Thomas Tauris for discussions on nearby neutron stars. We thank Lars Bergström and Gia Dvali for helpful comments on the manuscript.
[1] N. Arkani-Hamed, S. Dimopoulos and G. Dvali, Phys. Lett. B 429, 263 (1998) hep-ph/9803315.

[2] I. Antoniadis, N. Arkani-Hamed, S. Dimopoulos and G. Dvali, Phys. Lett. B 436, 257 (1998) hep-ph/9804398.

[3] N. Arkani-Hamed, S. Dimopoulos and G. Dvali, Phys. Rev. D 59, 086004 (1999) hep-ph/9807344.

[4] T. Han, J. D. Lykken and R. Zhang, Phys. Rev. D 59, 105006 (1999) hep-ph/9811350.

[5] G. F. Giudice, R. Rattazzi and J. D. Wells, Nucl. Phys. B 544, 3 (1999) hep-ph/9811291.

[6] L. J. Hall and D. R. Smith, Phys. Rev. D 60, 085008 (1999) hep-ph/9904267.

[7] S. Hannestad, Phys. Rev. D 64, 023515 (2001) hep$\mathrm{ph} / 0102290$.

[8] S. Cassisi, V. Castellani, S. Degl'Innocenti, G. Fiorentini and B. Ricci, Phys. Lett. B 481, 323 (2000) astro$\mathrm{ph} / 0002182$.

[9] S. Cullen and M. Perelstein, Phys. Rev. Lett. 83, 268 (1999) hep-ph/9903422.

[10] V. Barger, T. Han, C. Kao and R. J. Zhang, Phys. Lett. B 461, 34 (1999) hep-ph/9905474.

[11] C. Hanhart, D. R. Phillips, S. Reddy and M. J. Savage, Nucl. Phys. B 595, 335 (2001) nucl-th/0007016.

[12] C. Hanhart, J. A. Pons, D. R. Phillips and S. Reddy, Phys. Lett. B 509, 1 (2001) arXiv:astro-ph/0102063.

[13] S. Hannestad and G. Raffelt, Phys. Rev. Lett. 87, 051301 (2001) hep-ph/0103201.

[14] N. Kaloper, J. March-Russell, G. D. Starkman and M. Trodden, Phys. Rev. Lett. 85, 928 (2000) hhep$\mathrm{ph} / 0002001$.

[15] G. R. Dvali, G. Gabadadze, M. Kolanovic and F. Nitti, Phys. Rev. D 64, 084004 (2001) hep-ph/0102216.

[16] H. Tananbaum, IAU Circ. No. 7246 (1999).

[17] D. J. Thompson et al., Astrophys. J. Suppl. 101, 259 (1995). D. J. Thompson et al., Astrophys. J. Suppl. 107, 227 (1996). R. C. Hartman et al., Astrophys. J. Suppl. 123, 79 (1999).

[18] J. A. Esposito, S. D. Hunter, G. Kranbach and P. Sreekumar, Astrophys. J. 461, 820 (1996).

[19] F. M. Walter, S. J. Wolk and R. Neuhäuser, Nature 379, 233 (1996); F. M. Walter and L. D. Matthews, Nature 389, 358 (1997); F. M. Walter, Astrophys. J. 549, 433 (2001).

[20] T. M. Tauris et al., Astrophys. J. Lett. 428, L53 (1994).

[21] J. D. Pilkington et al., Nature 218, 126 (1968).

[22] M. I. Large, A. E. Vaughan and R. Wielebinski, Nature 220, 753 (1968).

[23] S. Johnston et al., Nature 361, 613 (1993).

[24] See for instance the URL www-glast.stanford.edu

[25] G. G. Pavlov, G. S. Stringfellow and F. A. Cordova, Astrophys. J. 467, 370 (1996).

[26] M. B. Larson and B. Link, Astrophys. J. 521, 271 (1999). 